\title{
Programmatische Stichworte zum Selbstverständnis der Kirchenfunkarbeit
}

von Meinhard Schmidt-Degenhard

\section{Zur Fragestellung}

"Der liebe Gott hat eine gute Herberge in einem Hörfunkhaus“, bemerkte 1972 der damalige WDR-Hörfunkdirektor Fritz Brühl mit Blick auf jene Programmkästchen im Funk, die den Themen Religion, Kirche und Theologie zugedacht sind ${ }^{1}$. Ob diese Herbergen in Funk und Fernsehen wirklich so gut und ziemlich sind, wag(t)en immer wieder Kritiker aus Kirchenkreisen zu bezweifeln. Denn verantwortlich für den $\mathrm{Zu}$ stand und das Interieur dieser Herbergen sind, abgesehen von den Kirchen mit ihren Gottesdienst- und Andachtsangeboten, vor allem die Fachredaktionen im Funk.

Wie aber verstehen die dort arbeitenden Journalisten und Redakteure ihre Aufgaben? Sehen sie sich als Sachverwalter öffentlicher Kommunikation oder als Hausmeister einer göttlichen Herberge, um im Bild zu bleiben - oder beides? Es stellt sich also die programmatische Frage nach einem möglichen theologisch-journalistischen Selbstverständnis der Kirchenfunkarbeit. Die Beschäftigung mit dieser Problematik im Rahmen meiner theologischen Diplomarbeit hat gezeigt, daß sich mit Recht ein spezifisches Eigenverständnis der Kirchenfunkarbeit behaupten läßt $\mathrm{t}^{2}$; wenngleich doch keinesfalls in diesem Zusammenhang von dem Selbstverständnis der Kirchenfunk-Programmacher gesprochen werden darf. Es lassen sich ,nur' einzelne ansatzhafte Stichworte ausmachen, die je verschieden von den jeweiligen Redakteuren übernommen werden.

Die großen Theoriediskussionen über das, Wie ${ }^{\mathfrak{c}}$ und ,Was ${ }^{\boldsymbol{k}}$ des Kirchenfunks gehören zwar eher der Vergangenheit an (vieles von damals ist heute selbstverständlich) dennoch scheint eine gelegentliche Reflektion über diesen journalistischen Arbeitsbereich, über seine Möglichkeiten, Chancen und Verantwortungen mehr als wichtig. Dies soll im folgenden versucht werden. Es geht darum, ausgehend von den Ergebnissen meiner Arbeit, in eigener Akzentuierung programmatische Stichworte in Erinnerung zu rufen, die unverzichtbar erscheinen für dieses Feld christlicher Publizistik.

\section{Zur Frage eines Selbstverständnisses der Kirchenfunkredaktionen}

Die von mir im Rahmen meiner Untersuchung durchgeführten Befragungen einzelner Redakteure zu ihrem theologisch-journalistischen Selbstverständnis und die Durchsicht der vorliegenden Literatur haben gezeigt, $\mathrm{da}$ es bei den einzelnen Redakteuren(innen) verschiedene Arten der Zuordnung von Journalismus und Theologie gibt. Teilweise recht krasse Unterschiede traten hervor bei der Frage nach Bedeutung bzw. Inhalt ihres theologischen Selbstverständnisses. Eine erste wichtige Feststellung ist, daß die befragten Redakteure sich vor allem und zuerst als Journalisten verstehen und

Meinhard Schmidt-Degenhard studierte Philosophie, Theologie und Pädagogik in Frankfurt und Utrecht/NL und schloß seine Studien mit einer Diplomarbeit zum Thema Kirche und Rundfunk ab. Er ist Mitarbeiter in Erwachsenenbildung und freier Journalist. 
darauf Wert legen, sich den gleichen journalistischen Kriterien zu unterwerfen wie Kollegen anderer Fachredaktionen. Darüber hinaus läßt sich bei der Mehrheit von ihnen ein besonderes Verhältnis zu der von ihnen bearbeiteten Thematik ausmachen, von dem her sie sich bewußt in diesem Bezugsfeld ,Kirche - Rundfunk - Gesellschaft ${ }^{*}$ engagieren. Bei einer kleineren Gruppe wurde nicht allein ein theologisches Eigenverständnis, sondern ein christliches, ja sogar engagiert kirchliches Verständnis der eigenen Programmarbeit erkennbar.

So war für die eine Gruppe das entscheidende Selbstverständnis ihrer Arbeit immer ein journalistisches, das sie strikt trennen von ihrer eigenen kirchlichen oder theologischen Position. Man erhebt für die Programmarbeit keinen Anspruch auf ein theologisches Selbstverständnis. Sein durchaus vorhandenes und bewußßtes Sachengagement sieht einer dieser Redakteure denn auch nicht in seiner Kirchengebundenheit begründet, sondern leitet es her vom Programmauftrag ,Bildung c der öffentlich-rechtlichen Medien. Er sieht sich nicht einer strikten Neutralität verpflichtet, sondern versteht sich in Berufung auf das Bundesverfassungsgericht als "Faktor der Meinungsbildung“3. Denn würden sich die Journalisten aus dem Geschäft der Meinungsbildung heraushalten, so blieben - vergleichbar einem Monopol - als die einzigen Sachvermittler der christlichen und religiösen Dimension die Vertreter der institutionellen Kirchen.

Demgegenüber beschreibt ein anderer Redakteur von seinem theologischen Selbstverständnis her seine Arbeit als „Brückenbau“ - als ein Bemühen, „Kirchliches Unkirchlichen nahezubringen “. Eine Trennung zwischen beruflicher und persönlicher Ebene lehnt er daher auch ab. Ein ihm gedanklich verwandter Kollege ordnet die Elemente des theologischen und journalistischen Selbstverständnisses mit den Worten „inhaltlich und handwerklich" einander zu. Den Journalismus sieht er im Dienste der Theologie als schon "sehr klar ausgerichtet auf letzten Endes Verkündigung“. Wenn man auch deutlich Wert legt auf eine fachlich-exakte journalistische Arbeit, so weigert man sich doch, zu unterscheiden zwischen Beruf und persönlichem Christsein. Ein anderer Vertreter dieser sich besonders stark aus katholischen Vertretern rekrutierenden Gruppe von Redakteuren sieht sein journalistisches Verständnis stark von theologischen, und das heißt für ihn vor allem von ethischen Elementen durchzogen. Für seine Programmarbeit ist es ihm wichtig, sich nicht nur „allgemein religiös, sondern am konkreten kirchlichen Glauben zu orientieren “. Sein Versuch, Lebenshilfe und Sinndeutung anzubieten, ist ausgerichtet an den „Lösungsmöglichkeiten aus der Sicht des Christentums und der Kirche“.

\section{Stichwort: öffentlich-rechtliches Medium}

Eine ganz wesentliche und nicht nur zufällige Rolle bei der Frage nach dem Selbstverständnis der Kirchenfunkarbeit spielt der Aspekt des öffentlich-rechtlichen Mediums, nicht nur insofern er die faktische Arbeitssituation beschreibt, sondern insofern damit die besondere Chance und Verpflichtung einer theologischen Journalistik angesprochen ist in nicht-kirchlicher Organisation und für nicht primär kirchlich sozialisiertes Publikum. Speziell auf die Frage, was ihnen dieses Stichwort bedeute, betonte die Mehrheit der befragten Redakteure die damit verbundene besondere Verpflichtung und Verantwortung, in Unabhängigkeit von den Kirchen und ausgerichtet an einem alles andere als kirchlich-frommen Rezipientenkreis die religiös-kirchliche Dimension im Funk abzudecken. Entscheidendes Gewicht besitzt dabei die bereits erwähnte Leitlinie öffentlich-rechtlicher Journalistik, nämlich „Medium und Faktor der öffentlichen 
Meinungsbildung “ zu sein". Zielt das erste Stichwort ,Medium auf Vermittlung, auf den Forumsgedanken ab, so meint ,Faktor' über diese Vermittlungsaufgabe hinaus (oder daneben) die Rolle des Journalisten als eines aktiven Partners im Prozeß der Meinungsbildung.

Kirchlicherseits (aber nicht nur dort) wird bei der Behandlung umstrittener Themen besonders gern auf die Vermittlungsrolle des Journalisten verwiesen. Dagegen ist kritisch zu fragen, ob so Journalisten nicht Gefahr laufen, zu „Marionetten“" zu werden, „die auf einer Bühne auftreten, hinter deren Kulissen die gesellschaftlichen Gruppen

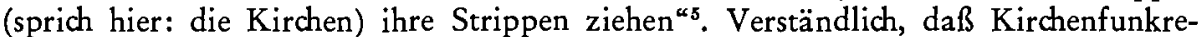
dakteure einer "Spiegeltheorie" entgegentreten, die die vornehmliche journalistische Aufgabe in einer Widerspiegelung kirchlicher bzw. gesellschaftlicher Gesprächsinhalte sieht. Fragt man ideologiekritisch nach dem Nutzen einer rein auf Vermittlung ausgerichteten journalistischen Position, so wird deren Gefährlichkeit deutlich. Dieser Vermittlerthese müsse man allein schon deswegen mißtrauisch gegenüber treten, so warnte ein Kirchenfunkredakteur im Gespräch, weil sie die, die sie vertreten, so stark begünstigt.

Eine Unterstützung findet ein derartiges journalistisches Selbstverständnis, das sich auch als Faktor der Meinungsbildung sieht, im übrigen in der päpstlichen Pastoralinstruktion "Communio et Progressio“, heißt es doch dort: "Die Kommunikatoren sind Anwalt und Stimulator im Gespräch der Gesellschaft" ${ }^{\text {" }}$. Ein bloßes Widerspiegeln und Vermitteln gesellschaftlicher und (auf die Kirche bzw. den Kirchenfunk bezogen) kirchlicher Gegebenheiten würde den Programmauftrag des öffentlich-rechtlichen Systems verfehlen, und wäre zudem (kirchen-)politisch gefährlich.

Im übrigen: geht es wirklich um die Vermittlung und Erörterung relevanter religiöser und kirchenpolitischer Dimensionen, so darf sich dies in den wenigsten Fällen auf die institutionelle Seite der Kirchen beschränken. Denn, so ein Kirchenfunkredakteur, „ein Großteil der kulturellen, der religiösen Kräfte entzieht sich ja organisatorischen Kriterien, sie sind aber wichtig für das Gespräch, für die Frage der Lebensorientierung ${ }^{\star}$. Folglich könne man nicht davon sprechen, daß die Kirchen bzw. die Vertreter der Kirchenleitungen das, was religiös relevant ist, in sich vollständig zum Ausdruck bringen können; denn sie haben schließlich ein bestimmtes Mandat und eine bestimmte Optik.

Gerade aber die gelegentlichen kommunikationspolitischen Machtansprüche einzelner Kirchenleitungen gegenüber dem Funk führen zu gewissen, in ihren Strukturen immer gleichen Konflikten. So verständlich der Wunsch nach Selbstdarstellung seitens der Kirchenleitungen ist, theoretisch legitimiert durch die ,Vermittlerthese', so sehr sind doch die Kirchenfunkredaktionen nicht nur zur Vermittlung der herrschenden religiösen und kirchenpolitischen Kräfte verpflichtet. Gerade mit Blick auf die Artikulationsmöglichkeiten offizieller Kirchenleitungen (PR-Arbeit, Presseorgane usw.) kommt es dem Funk zu, statt Ausgewogenheit in der Vermittlung bewußt Akzente zu setzen und Kommunikationsgerechtigkeit zu praktizieren gegenüber relevanten, aber nicht hinreichend organisierten Kräften?

\section{Stichwort: Information}

Den wichtigsten Aspekt der eigenen beruflichen Arbeit bildet für die meisten der befragten Redakteure die Informationsvermittlung. Information stellt die Grundbedin- 
gung jedweder aktiven Lebensgestaltung dar. Informationen über die verschiedensten Aspekte der den einzelnen umgebenden Mit- und Umwelt ermöglichen erst jene Orientierung, die Voraussetzung zielbewußten Handelns ist. Das Programm, auch in der Sparte Kirchenfunk, steht im Dienst des Hörers, hat ihm Orientierung zu vermitteln, denn „nur so ist er wirklich dabei, wo es um die Formung des wirtschaftlichen, politischen, gesellschaftlichen, menschlichen und religiösen Lebens seiner Zeit geht ${ }^{*}$ (CeP, Nr. 34). Dies setzt aber mehr voraus als eine reine Nachrichten wiedergabe.

Informationsbeschaffung und -weitergabe haben immer gesellschaftliche Ausrichtung, haben gesellschaftliche Wirkungen. Jeder Programmacher in den Massenmedien übt von da aus "einen hochpolitischen Beruf aus, ganz gleich, in welcher Redaktion er arbeitet", gibt der ehemalige Kirchenfunkredakteur Manfred Linz zu bedenken ${ }^{8}$. Für den Kirchenfunk bildet die institutionelle Wirklichkeit von Kirche einen ersten Gegenstand der Information. Hinzu kommt aber (und das deckt sich keinesfalls mit dem ersten) die Information über die Verkündigung einer Botschaft, des Evangeliums. Es geht um die Informationsvermittlung über das geschichtliche Konkretwerden der Botschaft in Kircheninstitutionen und -strukturen, in Basisgruppen und Gemeinden, in politischen Handlungsmodellen und sozialen Aktivitäten. Dabei besitzt Information Rückwirkungen auf das, worüber informiert wird. Für das Beispiel Kirche heißt dies: sie konfrontiert unbarmherzig mit der in der Vergangenheit lange Zeit außen vor gebliebenen Offentlichkeit. „Information verweigert der Verkündigung und dem Glauben im Bereich des nur Gedachten zu verharren und sich darin zu verflüchtigen $^{\text {"g. }}$. Jene Grenzen sind gesprengt, die christliche Information in das Korsett kirchlich-institutioneller Geschehnisabläufe hineinzwängen wollen. Informationsvermittlung aus christlicher Sicht, aber auch Informationsvermittlung über christliche Verkündigung und ihr Konkretwerden haben nicht mehr nur die Kirche zum Objekt ihrer Arbeit, sondern auch all die persönlichen Situationen und gesellschaftlichen Ereignisse, in denen Menschen auf eine Botschaft der Befreiung und Erlösung hoffen ${ }^{10}$.

Wenn Kirche von ihrem eigenen theologischen Selbstverständnis nicht für sich selbst lebt, sondern für die Welt, dann folgt daraus, daß es für die Praxis der Informationsvermittlung nur konsequent ist, weniger über Christliches zu berichten als vielmehr dristlich über anderes. Information im Sinne dieser "Guten Nachricht" ist aber nur die Information, die etwas bewirken will, die abzielt auf eine Abänderung „schrankenloser Selbstbehauptung der einzelnen " und „souveräner Staaten gegeneinander" (H.-E. Bahr). Das heißt: will Informationsvermittlung Kommunikation im und Partizipation am Prozeß individueller und gesellschaftlicher Befreiung ermöglichen, so wird sie mehr sein müssen als nur Faktendarstellung und -aneinanderreihung.

Information ist allein schon vom Informationsvermittler her keine neutrale Weitergabe, und sie darf es nicht mehr sein im Sinne der Brecht'schen Mahnung: „Wer nichts weiß und nicht handelt, der ist ein Dummkopf. Wer aber etwas weiß und nicht handelt, der ist ein Verbrecher!" Ein Blick auf die Inhalte heutiger Informationssendungen, die uns mit den Gefahren der atomaren Aufrüstung, brennender sozialer Fragen und der Zerstörung unseres Lebens(raumes) konfrontieren, zeigt, daß Information nicht mehr neutral bleiben kann und darf (sehr wohl aber sachlich, fair und engagiert $)^{11}$. Die Illusion einer sich außen vorhaltenden Informationsvermittlung ist zerstört (und das nicht erst seit heute), erst recht für die Informationssendungen einer Kirchenfunkredaktion, die oft mehr als Zeitfunkredaktionen die Chance haben, einzelne Informationen gezielt aufzuarbeiten und Betroffenheit zu wecken. Diese Unausweichlichkeit von Subjektivität und Engagement darf allerdings nicht entbinden von der Pflicht zur Sachlichkeit, vom „Stil der Information"12. Konsequente Folge dieses 
Stils der Information müßte eine Aufklärung des Rezipienten sein, die in Erinnerung ruft, daß im Sinne christlicher Informationsvermittlung die Aufnahme einer Nachricht nicht mit ihrer Entgegennahme abgeschlossen ist, daß im Gegenteil der entscheidende Prozeß dann erst beginnt. „Wir müssen antwortend auf sie eingehen . . . wir sind nicht erst im Handeln an der Welt einer des anderen Schuldner, wir sind es bereits im Erkennen der Welt"13. Für den Rezipienten stellt sich dann schließlich die Frage: ,Was mache ich mit dem Gehörten/Gesehenen, was kann ich machen?` Auf die Dauer wird sich für eine Programmarbeit, die mehr als nur Nachrichten weitergeben will, auch die Frage stellen, wie man dabei mehr als bisher dem Hörer oder Zuschauer Hilfestellung leisten $\mathrm{kann}^{14}$.

\section{Stichwort: Verkündigung}

Information kann, ob gewollt oder ungewollt, zur Verkündigung werden, zur Verkündigung der Nachricht. Damit stellt sich die Frage, wie es für ein theologisch-journalistisches Selbstverständnis um diesen Begriff der Verkündigung bestellt ist. Auf der formalen Ebene bzw. zur juristischen Unterscheidung trennt man Verkündigungssendungen von redaktionellen Beiträgen. Dennoch erübrigt sich damit auf der Ebene der Selbstverständnisdiskussion noch nicht die Frage nach einem „indirekten Verkündigungsimpetus" der Redaktionen. Interessanterweise findet sich weder in der Literatur noch in den Außerungen der befragten Redakteure eine ausreichende Definition dieses Begriffs der ,indirekten Verkündigung', wie er früher oft in entsprechenden Diskussionen gebraucht wurde ${ }^{15}$. Die eigene Arbeit wird von vielen Redakteuren sehr wohl als Verkündigung gesehen, denn schließlich mache man dies, so ein Redakteur, „nicht aus irgendeinem neutralen Interesse heraus!“ In gleicher Richtung meinte ein Kollege, daß Kirchenfunkarbeit immer eine Art von Verkündigung ist. Heute könne man durch eine einfache Information über Sinn mehr Sinn vermitteln, „als wenn ich etwa eine Stunde über ,Sinn" mache“.

$\mathrm{Daß}$ de facto so etwas wie Verkündigung auch in den redaktionellen Beiträgen überkommt, kann mit dem Wortpaar ,direkt - indirekt' nicht annähernd erklärt werden - was meint im übrigen das Wort ,direkt'? Sehr viel realistischer scheint die Unterscheidung zwischen amtskirchlich-autorisierter und nicht-amtskirchlich-autorisierter Verkündigung, zwischen Amt und Laie zu sein (was im übrigen eine Aufwertung der Funktion des Laien in Theologie und Kirche bedeutet). In den Redaktionen steht im Vordergrund die journalistische Arbeit, die allerdings zweifelsohne eine „inhärente theologische Qualität" besitzt ${ }^{16}$. Diese theologische Qualität, die nicht bestritten werden soll, ist jeweils abhängig von Personen und Persönlichkeiten. Den meisten Redakteueren geht es darum, a) sich immer wieder als sachliche Vertreter ihres Berufsstandes und nicht als Prediger verstanden zu wissen, und b) sich angesichts der ohnehin oft genug beobachtbaren Macht- und Einflußgelüste der Kirchenleitungen auf die Redaktionen zumindest formal aus dem "Geschäft der Verkündigung" herauszuhalten. Darüber hinaus dürfte allerdings für beide Seiten die Frage wichtig sein, wo aufgrund der medialen Bedingungen die Grenzen der Verkündigung zu ziehen sind. Diese in den "Gründerjahren" bereits geführte Diskussion findet heute ihre aktuelle Weiterführung in den Debatten um das kirchliche Engagement in den "Neuen Medien“. Die Erwartungen an die Medien im Bereich der Verkündigung gilt es einer erneuten Realitätskontrolle zu unterziehen, um auf dem Weg in die neue Medienzukunft (oder das, was man dafür hält) nicht verlockenden Illusionen und gefährlichen Trugbildern auf- 
zusitzen, denen die theologische (und nicht zuletzt die finanzielle) Ernüchterung nur $\mathrm{zu}$ rasch folgen wird.

\section{Für eine Ent-Privatisierung der Verkündigung}

$\mathrm{Ob}$ nun praktiziert durch autorisierte Sprecher der Kirchen oder durch entsprechend engagierte Journalisten, in jedem Fall bekommt die Wort- bzw. Bildverkündigung via Medien eine neue Dimension, die Dimension der Offentlichkeit. Dies meint aber nicht nur die faktische Offentlichkeit im Sinne des stärker öffentlichen und damit weltlichen Publikums. Angesprochen ist damit vielmehr die Rückwirkung auf Inhalt und Konzept einer Verkündigung, die nun nicht mehr auf innerkirchliche Unverbindlichkeiten sich zurückziehen kann, sondern die nun den Anspruch und die Erwartungen einer Offentlichkeit auf ein für das Ganze politischer und sozialer Wirklichkeit relevantes Sprechen einzulösen hat.

Theologischerseits wurde bereits sehr früh auf die Notwendigkeit eines entprivatisierten Sprechens von Gott in den Medien hingewiesen. So gab Johann Baptist Metz 1968 in einem Vortrag vor der ,Katholischen Rundfunk- und Fernseharbeit Deutschlands zu bedenken: $\mathrm{Zu}$ lange „wurde das Verständnis des Verkündigungsgeschehens von vornherein privatisiert und gewissermaßen existentiell intimisiert. Das Verkündigungswort wurde als reines Anredewort, nicht aber als gesellschaftsbezogenes VerheiBungswort" verstanden; denn, so folgert er, "gerade um die Existenz zu treffen, kann heute nicht rein existentiell gesprochen werden "17.

Kirchenfunkprogramme haben in konsequenter Weiterführung dieses Gedankens nicht in erster Linie über Kirchen zu berichten, sondern über persönliche und gesellschaftliche Erfahrungen von Leid und Freude, Hoffnung und Angst, denn Kirche und ihre Verkündigung existieren ,pro mundo, „um der Pro-Vokation des Heils willen "18. Gibt es so etwas wie einen latenten Verkündigungsimpetus innerhalb der Kirchenfunkredaktionen, so wird dieser angesichts einer grundsätzlich öffentlichkeitsbezogenen Arbeit nur als entprivatisierte Verkündigung verantwortbar sein.

Nun zeigt die wiederholt vorgetragene Kritik an bestimmten gesellschaftsbezogenen Beiträgen der Kirchenfunkredaktionen, daß der Schritt zu einer Entprivatisierung der Verkündigung in manchen kirchlichen Kreisen noch nicht geleistet ist. Was Kirchenvertreter gelegentlich als Politisierung und „Entkirchlichung" beklagen, spielt mittlerweile im Selbstverständnis der Redaktionen (wie Gespräche und Literatur zeigen) eine ganz wichtige Rolle. Die Verpflichtung einer Offentlichkeit gegenüber zwingt zum Herausarbeiten der für die Gesellschaft relevanten Inhalte christlicher Botschaft. Dabei würde eine bei einigen Kirchenkreisen anfänglich Zustimmung hervorrufende $\mathrm{Be}$ schränkung auf den ,homo privatus et religiosus' die Kirche samt ihrer Verkündigung letztlich in die Funktion einer Entlastungsinstanz für den einzelnen und die Gesellschaft drängen, „der man aber keine gesellschaftskritische und gesellschaftsutopische Kraft mehr zutraute ${ }^{\alpha 19}$.

Der Ruf nach einer entprivatisierten Verkündigung will daran erinnern, daß eine wirklich, direkte ${ }^{6}$ Verkündigung nur die sein kann, die direkt an gesellschaftlicher, an menschlicher Wirklichkeit orientiert ist. Ohne damit die Präferenz einseitig auf die rein gesellschaftliche Ebene lenken zu wollen, gilt es, um die strukturelle Verwobenheit von Hoffnungs- und Rechtlosigkeit, von Verzweiflung und Unterdrückung, von Angst und Macht, von Sein und Bewußtsein zu wissen ${ }^{20}$. 


\section{Stichwort: Kirche}

Trotz oder gerade wegen der offenkundigen Bedeutung, die dem Stichwort ,Kirche im Rahmen der Redaktionsarbeit zukommt, scheint es interessant, einmal zwei besondere Gesichtspunkte zu beleuchten, wie die Kirche(n) in der redaktionellen Arbeit vorkommt: zum einen der Aspekt des (nicht zuletzt auch persönlichen) Verhältnisses der Programmacher zur Kirche, und zum anderen die Bedeutung von Kirche als Objekt journalistischer Arbeit.

\subsection{Kirchenge- bzw. -verbundenheit}

Die bisherigen Ausführungen haben bereits die entscheidende Rolle erkennen lassen, die das eigene Christsein, ihre Identifikation mit bzw. ihr Engagement in ihren Kirchen für einige Redakteure und für ihr berufliches Selbstverständnis spielen. Divergierende Positionen werden dabei deutlich: so legen die einen, angesprochen auf die Frage ihrer Kirchenge- bzw. -verbundenheit, bewußt Wert auf eine grundsätzliche Trennung der persönlichen Kirchenmitgliedschaft von ihrer Funkarbeit. Ein Ineinanderspielen beider Rollenfelder lehnen sie ab. Dem entgegengesetzt der Ansatz einiger anderer Redakteure, die ihrer Kirchenverbundenheit einen ganz entscheidenden Stellenwert für ihre Programmarbeit zum Stichwort ,Kirche einräumen. Sie verstehen sich $z$ war nicht als „Repräsentanten von Kirche im amtskirchlichen Sinne“, sehen sich aber als im Funk engagierte Kirchenmitglieder, wenn auch nicht im kirchlichen Auftrag. Die Spannung zwischen dem beruflichen Engagement und seiner Kirchengebundenheit möchte ein Redakteur nicht nur als Herausforderung für die Journalistik sehen, sondern auch als Prüfstein für seine persönliche Kirchenmitgliedschaft: „Ob ich will oder nicht: ich muß mich selbst fragen und vor allem fragen lassen von Kollegen und Hörern, wie es denn mit meinem Glauben, meinem Katholischsein bestellt ist $^{\alpha 21}$.

Interessanterweise, so zeigt ein Blick in die Gesprächsäußerungen und in die einschlägige Literatur, findet sich ein derartiges mehr oder minder stark betontes „sentire cum ecclesia" vornehmlich unter katholischen Redakteuren. $\mathrm{Ob}$ diese unterschiedlichen Selbstverständnisse in der Frage der Kirchengebundenheit einen direkten theologischinhaltlichen (sprich ekklesiologischen) Hintergrund haben, oder ob hier rein persönliche Faktoren eine Rolle spielen, läßt sich nicht auf den ersten Blick ausmachen. Ganz sicher kommt der eigenen kirchlichen und beruflichen Sozialisation eine nicht zu unterschätzende Bedeutung zu. Für das konkrete Beispiel der fünf Redakteure, die ich für meine Arbeit befragt habe, zeigt sich, daß von drei protestantischen Journalisten zwei vormals als Pfarrer im kirchlichen Dienst $\operatorname{standen}^{22}$, und die beiden katholischen Gesprächspartner vor ihrer jetzigen Redakteursarbeit in kircheninternen publizistischen Einrichtungen tätig waren. Lediglich einer der fünf kam nach einem reinen Fachstudium der Theologie (ohne ein kirchliches Amt je angestrebt zu haben) über die verschiedensten Stationen des Journalismus in den Kirchenfunk.

\subsection{Kirche als Objekt journalistischer Arbeit}

Entsprechend den deutlich verschiedenen Positionen in der Frage der persönlichen Kirchenverbundenheit treten auch divergierende Einschätzungen auf bei der Frage danach, was Kirche als Objekt journalistischer Arbeit bedeutet. Die eine Gruppe sieht sich in Folge ihres ,Mitfühlens mit der Kirche in einer besonders verantwortungsvollen Zwitterstellung zwischen Kirche und Welt. Man betrachtet es als seinen Auftrag, Kirche der Welt bzw. die Welt der Kirche zu vermitteln. Vermittlung heißt, „daß also 
da nicht nur ein neutrales Hinstellen mitgemeint ist, sondern der Versuch, das einander anzunähern."

Ein derartiges Selbstverständnis als Vermittler bzw. Dolmetscher findet kirchlicherseits (gerade im katholischen Raum) wenig Widerspruch. So bezeichnet etwa der im katholischen Bereich für die Medienarbeit verantwortliche Prälat W. Schätzler die Position des Kirchenfunkredakteurs als „Verkünder, aber auch kritischer Begleiter", - und spricht davon, daß die Programmacher „Mittler von religiös-kirchlichen Informationen und Mittler von in der Kirche vertretenen Meinungen " sind"

Gegen eine vorschnelle und unkritische Ubbernahme dieser bereits oben erwähnten Vermittlerthese setzen sich allerdings auch einige Kirchenfunkredakteure zur Wehr (s. o. 3.), zumal es sich dabei um eine kirchliche Machtpositionen stabilisierende Theorie handelt. Im Zusammenhang des Stichworts ,Kirche als Objekt der Arbeit' ist von kirchenoffizieller Seite (im katholischen Raum wohl vermehrt) der Vorwurf zu hören, offizielle Amtsvertreter würden zugunsten einer deutlichen Zunahme ,progressiver Stimmen aus den Programmen verdrängt ${ }^{24}$. Dahinter steckt nicht nur eine falsche Erwartungshaltung und Unkenntnis gegenüber den Medien, sondern die viel entscheidendere und grundsätzlichere Frage nach dem Verhältnis der Kirche zur Offentlichkeit; ja letztlich verbirgt sich dahinter die ekklesiologische Gretchenfrage nach dem, was eigentlich Kirche ist, wer Kirche bildet. Ist Kirche nur dargestellt und vollgültig ins Bild bzw. ins Wort gekommen, wenn in entsprechendem Maße Amtsträgern die Möglichkeit zur Außerung gegeben wurde, oder aber existiert Kirche auch (und gerade) in Projekten und Aktionen der Basis, in Außerungen und Meinungen einzelner Gläubiger und einzelner Gemeinden; ja lebt Kirche nicht gerade dort, wo zwei oder drei in seinem Namen leben und handeln, Glauben versuchen und $Z_{\text {weifel }}$ (an der Realität) wagen? Wenn daher (wohl immer wieder von neuem) die Forderung erhoben wird, in den Kirchenfunkprogrammen müsse "normale Kirche stattfinden "25 bzw. das dort vermittelte Bild müsse „mit der Wirklichkeit dieser Kirche“ übereinstimmen $^{26}$, so sollte dies Anstoß sein, grundsätzlich, d. h. theologisch, aber eben nicht nur institutionell und kirchenpolitisch, nach der ,Normalität' und Wirklichkeit von Kirche zu fragen.

Kirchenfunkarbeit versteht sich auch als bewußtes, kritisches Gegengewicht gegenüber einem religiös-christlichen Monopolanspruch der Groß- und Amtskirchen. Kirche, so ein Redakteur im Gespräch, „ist für uns ein gesellschaftliches Phänomen unter anderen geworden". Als gesellschaftlich relevanter Gruppe komme den Kirchen das Recht zu, ihre Stimme und das, was sie inhaltlich vertreten, im Programm wiederzufinden ${ }^{27}$. Das Phänomen Kirche wird vom Ansatz her gesehen als eines von mehreren möglichen Erscheinungsbildern der Dimension der Religion im menschlichen Leben. Konnten vor Jahren noch die Kirchen für sich beanspruchen, die allein entscheidenden Ansprechpartner für Fragen der religiösen Orientierung zu sein, so finden sich für ein derartiges Ansinnen heute keine ausreichenden Legitimationsgründe mehr. Eine mit dem Themenbereich ,Religion und Kirche' befaßte christliche Journalistik, die dank ihrer öffentlich-rechtlichen Organisation den Kirchen nicht mehr als anderen gesellschaftlichen Gruppen verantwortlich ist, stellt eine Beschneidung des Monopolanspruchs der Kirchen dort dar, wo es um die Fragen nach und die Interpretation von Religion, Kirche, Christsein und seinem gesellschaftspolitischen Auftrag geht. Innerkirchlich leistet dies seinen Beitrag zur „Entstehung dessen, was man ,mündige Gemeinde nennt, und dies möglicherweise durchaus gegen den Willen derer, die Kirchen und Gemeinden leiten "28. Außerkirchlich bietet sich eine derartige Funkarbeit als Forum an, um existentielle, soziale und gesellschaftspolitische Fragen aufzufangen in einem 
Freiraum, der zwar vom christlichen Horizont mit umgrenzt ist, aber nicht zwangsläufig kirchlich begrenzt ist.

Kirchenfunkarbeit ist nicht mehr ausschließlich ausgerichtet auf den kirchlich gebundenen und gelebten Glauben. „Sie wird ihn berücksichtigen, sie wird ihm gewiß Gelegenheit zur Selbstdarstellung geben ... Aber sie darf sich an ihm nicht ausrichten; denn dann würde sie gegen das Gebot der Unparteilichkeit verstoßen“, so ein ehemaliger Programmacher des Kirchenfunks ${ }^{29}$. Auf diesem Wege wird nur konsequent einem Monopolverlust der Großkirchen im gesamtgesellschaftlichen Bereich auch im Sektor der Medien als dem „Gespräch der Gesellschaft" Rechnung getragen.

\section{Stichwort: Stellvertretung}

Für ein theologisch-journalistisches Selbstverständnis läßt sich das Stichwort der ,Stellvertretung, der Gedanke des stellvertretenden Handelns und Sprechens für die Sprachlosen und Ohnmächtigen, zweifach legitimieren: zum einen stellt es eines der wichtigsten Prinzipien christlicher Publizistik überhaupt dar, zum anderen meint Stellvertretung eine nicht zuletzt auch für Kirche von ihrem Gründungsauftrag her wesentliche Komponente, die auf einem quasi indirekten Weg auch Bedeutung bekommt für jene journalistische Arbeit, deren Objekt Kirche ist ${ }^{30}$. Denn über Kirche zu berichten hieße dann u. a., über ihr stellvertretendes Sprechen und Handeln in der Welt zu berichten (oder dies anzumahnen!) und damit selber sich ,in Stellvertretung zu engagieren.

Nicht selten wurde in der Vergangenheit kirchlicherseits dort, wo es um die Vertretung eigener Interessen gegenüber dem Rundfunk ging, Stellvertretung zugunsten der Selbstdarstellung hintangestellt; dennoch erweist sich theologisch argumentiert Stellvertretung als einer der Grundpfeiler christlicher Botschaft. Das im Glauben Befreitsein von der Angst um sich selbst, um die eigenen Abgründe, Ängste und Fehler, eröffnet dem Christen den Blick auf die Lebenssituation anderer, ihn umgebender Menschen. Die "Freiheit des Christenmenschen" (und damit auch die aus der Freiheit gewonnene Gelassenheit der Kirche als Gemeinschaft eben dieser Befreiten) schenkt „die einzigartige Möglichkeit, für andere zu handeln: für Schwache, für Isolierte, für Ohnmächtige und für die geringsten Brüder ${ }^{431}$. Stellt sich überhaupt die Frage nach dem Spezifikum gerade christlichen (Stellvertreter-)Handelns, so ist dies eben in der vom Glauben geschenkten Freiheit zu sehen, die befreit ist von verkrampfter Angst um das eigene Ich bin zu einem vorbehaltlos möglichen Engagement für andere. Das Geschenk der Freiheit läßt Christen und Kirchen immer deutlicher ihre Bestimmung erkennen, "für die Welt da zu sein und in vielfacher Weise - in der Weise des ,An-Stelle-von und ,In-der-Zuwendung-für ${ }^{\circ}$ - den Dienst der Stellvertretung zu tun ... Es erwacht eine neue Solidarität mit der Welt im Namen der Freiheit, der Individualität, des

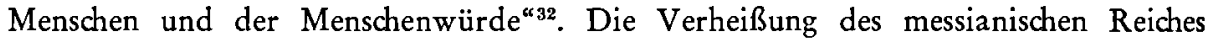
ebenso wie die Gnade des (biblisch gesprochen) Exodusgeschenks nimmt in die Pflicht zum Kampf für und zum Sprechen in Stellvertretung von Hoffnungs- und Rechtlosen, von Be- und Unterdrückten (wer auch immer das gerade in einer spätbürgerlichen Industrie- und Wachstumsgesellschaft wie der unsrigen sein mag). Allein dieser Dienst in Stellvertretung legitimiert jenen anderen "Gottes-Dienst ${ }^{\text {; }}$; denn, so formulierte es Dietrich Bonhoeffer in der Zeit des Nazi-Faschismus, nur wer für die Juden schreit, darf gregorianisch singen! 
Angesichts dieser theologischen Relevanz des Stellvertretungsaspektes sind jene Kritikerstimmen an Kirchenfunkprogrammen nur schwer nachvollziehbar, die in der Praktizierung dieses publizistischen Elementes eine Verflachung oder Politisierung christlicher Existenzweise in der Welt sehen. Stellvertretung führt zwangsläufig (und dies nicht im Widerspruch zu einer guten journalistischen Arbeit) zu Engagement und Parteinahme. Und Parteinahme scheint immer wichtiger zu werden als ein Eintreten bzw. Aufgreifen des Verschwiegenen, dessen, was als Sand im Getriebe das Räderwerk der vermeintlich selbstverständlichen Sachzwänge in Kirche und Gesellschaft stört. Ist das harmlos erscheinende (weil alltägliche) Verschweigen, jene alte Katechismussünde der ,Unterlassung, nicht die größte Gefahr christlicher Publizistik; und schließlich: „man kann auch verschweigen, indem man so redet, daß es keinem weh tut $^{\alpha 3 s}$.

Ein Kirchenfunkredakteur unterschied im Gespräch auf seine Programmarbeit bezogen zwei Aspekte des Stellvertretergedankens: zum einen eine thematische Stellvertretung, die (un-)bewußt vernachlässigte Informationen und Themen aufgreift, und zum anderen eine personelle Stellvertretung für Hoffnungs- und Rechtlose, für einzelne und Gruppen, die innerhalb der festgefügten kommunikationspolitischen Machtstrukturen nicht (oder nur am Rande) zu Worte kommen. Derart praktizierte Stellvertretung im Rahmen des Kirchenfunkprogramms, die sich um eine Offenlegung verdeckter und um eine Beschaffung unterdrückter (oder zurüickgehaltener) Meinungen bemüht, erweist sich beispielsweise mit Blick auf bestimmte kirchenpolitische Entwicklungen (bzw. Entfremdungen im Verhältnis zwischen Leitung und Basis) als dringend geboten für ein gesundes binnenkirchliches Informations- und Meinungsforum. Das könnte bedeuten, sich z. B. der Fragen nach innerkirchlicher Demokratie, nach Christen- bzw. Menschenrechten in der Kirche ebenso wie neuer theologischer Entwicklungen anzunehmen, sich vor allem zu bemühen um eine Konfrontation christlich-kirchlicher Denk- und Lebensidylle mit den Problemen der atomaren Aufrüstung und der wirklichen Friedenssicherung, mit den Fragen der Kernenergie und des quantitativen oder qualitativen Wachstums mit all seinen sozialen Problemen. Stellvertretung konkretisiert sich aber genauso in einer ,Stellvertretung für den einzelnen'; dies geschieht im Aufgreifen der Fragen von Angst, Leid, Tod, von Freude und Hoffnung, also jener Fragestellungen, die im Gespräch der Gesellschaft eines sachkompetenten Fürsprechers bedürfen, um damit zu thematisieren, was für alle bereits Thema ist.

So kann eine daran orientierte Programmarbeit stellvertretend wesentliche gesellschaftliche und politische Themen in den binnenkirchlichen Diskussionsraum einbringen. Andersherum formuliert sie aber auch stellvertretend im Gespräch der Gesellschaft die Anliegen und das Denken der Christengemeinden (und ihre Befreiungsbotschaft) und thematisiert somit wieder die in einer technokratischen Habengesellschaft verschütteten existentiellen Dimensionen menschlichen Lebens.

Im übrigen gewinnt das Stichwort der Stellvertretung seine aktuelle Bedeutung, ja Dringlichkeit (auch für Kirchenfunkprogramme), wenn man sich mit der Medienzukunft in unserem Lande auseinandersetzt. In einer zunehmend zergliederten und kommerzialisierten Medienlandschaft (BTX, Video, Kabel- bzw. Pay-TV, Privatfunk usw.) wird sich mit besonderer Schärfe die Frage nach der Kommunikationsgerechtigkeit und nach der Berïcksichtigung jener stellen, die weder an den Regietischen der Medien sitzen noch sich den Zugang dorthin erkaufen können. 


\section{Stichwort: Lebenshilfe}

Der Gedanke der Stellvertretung findet seine Ergänzung bzw. Weiterführung im Stichwort der Lebenshilfe, geht es doch hier darum, stellvertretend für den einzelnen in der Offentlichkeit, d.h. in den Medien, Ängste und Hoffnungen, Freude und Trauer aufzunehmen und Hilfe und Orientierung anzubieten. Diesem Gedanken der Lebenshilfe begegnet man in der konkreten Kirchenfunkarbeit und in den Diskussionsbeiträgen zum theologisch-journalistischen Selbstverständnis dieser Redaktionen verhältnismäßig häufig ${ }^{34}$. Ohne gleich auf die medienspezifischen Bedingungen (und damit Grenzen!) einzugehen, könnte Lebenshilfe im Vorfeld definiert werden als eine möglichst konkrete und existentiell betreffende Hilfestellung zum konstruktiv-kritischen Umgang mit einzelnen Alltagserfahrungen und den da heraus sich ergebenden Fragen; sie will auf diese Weise eine immer nur begrenzte und auf Motivation der Betroffenen ausgerichtete Hilfe zur geglückten und sinnvollen Existenz anbieten. Was auch immer eine ,geglückte und sinnvolle Existenz' ausmacht, für den einzelnen ist sie bestimmt durch das Gelingen von Liebe/Sexualität, Individualität/Sozialität, Freiheit/Verantwortung, Kreativität/Realitätsorientierung, Kommunikation/Vertrauensfähigkeit ${ }^{35}$.

\subsection{Der einzelne - die bedrohte und vergessene Minderheit}

Die Notwendigkeit einer Lebenshilfe in unserer heutigen Zeit wird dort am krassesten erfahrbar, wo es um die Ängste und Fragen des einzelnen geht. Verlassenheitsgefühle, Konkurrenzdruck, Gefühlsarmut und Orientierungslosigkeit werfen ihn auf sich selbst zurück und lassen ihn zur "gefährdetsten Minderheit in der heutigen Welt" wer$\mathrm{den}^{36}$. In diesem Kontext Lebenshilfe anzubieten, bedeutet für die Kirchenfunkarbeit, sich dieser bedrohten und leicht vergessenen Minderheit des Individuums besonders anzunehmen. Dies heißt, all die Gefühle (Hoffnungen und Ängste) aufzunehmen und in die Offentlichkeit hinein zu formulieren, die ansonsten in einer technokratischen Leistungsgesellschaft wie der unsrigen nicht mehr "salonfähig " sind. Lebenshilfe konkretisiert sich im Ernstnehmen und Eingehen auf eine Sprach- und damit Existenzdimension menschlichen Lebens, die konstruktiv auszudrücken heute immer mehr verlernt wird. Hier kommt Lebenshilfe zur Deckung mit dem Stichwort der Stellvertretung; geht es doch um ein stellvertretendes Aufgreifen der Ängste und Sehnsüchte des einzelnen in der Gesellschaft. Gegenüber einer politischen und sozialen Realität, die geprägt ist von Leistung und Potenzdenken, von Technokratie und Rationalität, können entsprechend konzipierte Funkangebote ein Forum bieten zum Anund Aussprechen von Gefühlen, Ängsten, Wünschen und Träumen; nicht aber, um auf diese Weise ein Auffangbecken individueller, Sehnsuchtsrinnsale ${ }^{c}$ zu schaffen, sondern um eben im konstruktiv-kritischen Aufnehmen dieser Dimension menschlichen Lebens einen (ersten kleinen) Beitrag zu einer geglückten Existenz dieser gefährdetsten Minderheit, des einzelnen (zu der wir ja alle gehören), zu leisten.

Ober dieses Aufgreifen einer bestimmten Sprach- und Existenzdimension hinaus wollen einige Redaktionsprogramme auch Lösungsmöglichkeiten aus der Sicht des Christentums und der Kirche aufzeigen und ihre Lebenshilfe gezielt unter den Horizont christlicher Sinnantwort stellen. So sieht etwa der ZDF-Redakteur Michael Albus Zweck und Ziel seines "Kontakte-Magazins" darin, "dem Zuschauer aufgrund der Erfahrung der modernen Seelsorge Wege zu zeigen, wie er seine persönlichen Probleme und Lebensfragen bewältigen kann ${ }^{437}$. Damit ist ein weiterer entscheidender Aspekt einer Lebenshilfe via Massenmedien angesprochen: das gezielte Aufzeigen von 
Modellen und Beispielen gelungenen Lebens. Einer Publizistik, die die christliche Botschaft und Kirche zu ihrem Arbeitsobjekt hat, und die sich nicht nur journalistisch, sondern auch theologisch versteht, kommt es $\mathrm{zu}$, „einerseits aufzuspüren und aufzudecken, wo gelitten wird (und Modelle gegen das Leiden und für dessen Bewältigung zu entwickeln), und andererseits aufzuzeigen und teilnehmen zu lassen, wo mit Freude gefeiert wird", wie es T. Stählin fomuliert ${ }^{38}$. Will man der Wirklichkeit individueller und gesellschaftlicher Existenz gerecht werden, so gehört dazu, auch einmal das ,Schon-Gelungene' herauszuarbeiten und evtl. neben die Desintegration des Lebens Modelle der Integration zu stellen (ohne damit gleich in Ausgewogenheit und Harmonisierung zu verfallen!). Ein Aspekt, der zwar seine Berechtigung findet in dem Gedanken der ,integrativen Funktion' der öffentlich-rechtlichen Anstalten ${ }^{38}$, der aber auch oft genug politisch im Sinne einer Verschleierung von Wirklichkeit mißbraucht wird.

\subsection{Wider ein privatistisches Verständnis von Lebenshilfe}

Wer sich auf die Suche macht nach Möglichkeiten und Hilfestellungen für ein geglücktes Leben, wird auch immer das vielfältige Hineingeflochtensein individueller Existenz in die gesamtgesellschaftlichen Abläufe zu berücksichtigen haben. Gerade eine hochindustrialisierte Gesellschaft wirkt mit ihrer Komplexität bis in die persönlichsten und intimsten Lebensbereiche des einzelnen hinein. Wenn dem öffentlich-rechtlichen Rundfunk eine ,integrierende Funktion' (s.o.) im weitesten Sinne zukommt, so könnte dies auch heißen, sich um eine Integration der verschiedenen Bereiche menschlichen Lebens zu bemühen, darum die ,res privata' und die ,res publica' in all ihren Spielarten aufeinander zu beziehen und dabei deutlich werden zu lassen, wie das eine das andere mitbestimmt. Damit kommt die strukturelle Verflechtung von Angst und Macht, von Hoffnung und Herrschaft, von Freude und Freiheit zur Sprache. Ein privatistisches Verständnis von Lebenshilfe würde demgegenüber niemals die Gesamtwirklichkeit annähernd in den Blick bekommen, und wäre damit auch nicht fähig, mehr als Befriedung und Harmonisierung zu erreichen. Auf die politischen Implikate einer privatistischen Konzeption braucht wohl nicht eigens hingewiesen zu werden.

Ganz sicher geht es nun nicht darum, das Individuum zugunsten gesamtgesellschaftlicher Erklärungsmodelle beiseite zu lassen (vgl. 8.1), aber wichtig scheint, bei dem zwangsläufig stärker individuellen Charakter des Stichwortes Lebenshilfe immer darum zu wissen, daß die dort angesprochenen Probleme einen gesellschaftlichen Hintergrund und vielleicht sogar Erklärungsgrund haben. Es gilt zu erkennen, daß heute anders gelitten (und gefeiert) wird in Partnerschaft und Familie als vor zwanzig Jahren, daß in einer Gesellschaft, die an die Grenzen des Wachstums stößt, anders nach Sinn und geglücktem Leben gef ragt wird als in einer Gesellschaft der Aufbaujahre.

Für eine Programmarbeit, z. B. die des Kirchenfunks, bedeutet dies, vor allem um diese historisch-gesellschaftliche Ebene und um die Gefahr der Verschleierung und Harmonisierung einer allzu naiven Lebenshilfepraxis zu wissen. Mit Blick auf das, was Lebenshilfe via Massenmedien überhaupt erreichen kann, stellt sich die Frage nach einer Lebenshilfe als "Aufklärung der arcana imperii (Herrschaftskritik) ${ }^{* 40}$. Wenn Menschen in und (in irgendeiner Hinsicht immer auch) an dieser Gesellschaft leiden, so hat Lebenshilfe den Betroffenen durch Informationen und durch ein ,Zu-Worte-kommen-Lassen' Einsicht (oder besser Durchsicht!) in ihre Schwierigkeiten und deren Hintergründe zu ermöglichen, um vielleicht damit einen ersten Schritt hin zu einem konstruktiven, weil bewußten Umgang mit ihrer Situation zu versuchen. 
Demgegenüber „strittig ist die Rote-Kreuz-Vorstellung, die den Bürger qua Lebenshilfe wieder da hinein integrieren will, woraus er ja gerade durch Krankheit oder andere modi der Selbstdistanzierung herausgegangen ist ${ }^{\star 41}$. Es geht sehr wohl um ein Trösten, das hilft, mit Leiden umzugehen, - aber eben nicht um ein Ver-Trösten. Die Programmacher können ihren Beitrag leisten, um Leiden (und in anderer Hinsicht auch Freude) öffentlich zu machen, um damit einen ersten Anstoß zur öffentlichen Solidarisierung der Betroffenen zu geben. Solange nur jeder glaubt, ihm allein gehe es so oder ähnlich, wird sich an den Ausgangspositionen nichts ändern.

Hinzu kommt, daß ein derartiges Offentlichmachen von Leid und Freude, von Hoffnung und Herrschaft ein wichtiger Schritt ist, um dem Individualismus entgegen zu wirken, den wir mit unseren Massenmedien geradezu provozieren (jedem sein eigenes Ohr/Auge zur Welt in seinen eigenen vier Wänden). Bei alldem müssen aber gleichzeitig auch die Grenzen einer Lebenshilfe im Funk realistisch gesehen werden, da nicht selten dem Rundfunk Aufgaben zugeteilt werden, die zu bewältigen nicht ihm, sondern der Gesamtgesellschaft zukommt. Denn die Erfahrungen zeigen, daß Hörfunk und vor allem Fernsehen in ihrer für manchen Rezipienten magischen Anziehungskraft oft die letzte Hilfe und Seelentröstung bieten müssen.

\section{Stichwort: Die Frage nach der Lebensrelevanz}

Auch wenn sich dieser Apekt in dieser Formulierung nur selten in der einschlägigen Literatur findet, so berechtigt doch seine Bedeutung zu einem wenigstens kurzen Eingehen auf diesen für ein theologisch-journalistisches Selbstverständnis m. E. wesentlichen Gedankens. Das Stichwort der Lebensrelevanz kann als Weiterführung der in den 60er Jahren von Manfred Linz engagiert vorgebrachten Programmkonzeption eines "Kommentars zum Leben" gewertet werden ${ }^{42}$. Ging es damals um den Versuch, Religion als existentiell bedeutungsvollen Kommentar zum Leben des einzelnen und der Gesellschaft herauszuarbeiten und auf diesem Weg Orientierungshilfe zu bieten, so geht es heute um das öffentliche Fragen und Suchen nach dem, was in einer zunehmend komplexeren Gesellschaft wirklich Bedeutung, sprich Lebensrelevanz besitzt. Damit wird die direkte Verbindung zum vorherigen Stichwort der Lebenshilfe erkennbar.

Es geht darum, öffentlich prononcierte und provozierende Fragen nach der Lebensrelevanz zu stellen, Fragen, die so nicht oder zu wenig in den anderen Sparten der öffentlich-rechtlichen Medien gestellt werden ${ }^{43}$. Die Realität soll hinterfragt werden auf das hin, was eigentlich noch wichtig ist. „Was zählt denn eigentlich? Ist denn alles, was wir tun - ist es das denn eigentlich? ... Was ist überhaupt ein erfülltes Leben? ... Daß die Frage nach dem Glück, nach der Lebenserfüllung qualifizierter gestellt wird, auch das ist eine Aufgabe, die eine Religionsredaktion hat ${ }^{\star}$, so formulierte es ein Redakteur im Gespräch. Hinter alldem steckt das Bewußtsein, wie wichtig es ist, in einer Gesellschaft, die in weiten Teilen dem normativen Diktat des Faktischen unterworfen zu sein scheint, bestimmte kritische Fragen nach der Eigentlichkeit personaler und gesellschaftlicher Existenz wachzuhalten.

Nach der Lebensrelevanz zu fragen stellt einen (den?) unverzichtbaren Beitrag christlicher Botschaft für die Gesamtgesellschaft dar. Immer wieder radikal, d. h. nach der Wurzel fragend, Kritik am Bestehenden zu äußern ist eine zutiefst christliche Sache, stellt doch nichts jedwede Realität so sehr in Frage wie der christliche Zukunftsentwurf einer himmlischen Welt ${ }^{44}$. Als im August 1968 fremde Truppen die Tschecho- 
slowakei überfielen, schob sich am ersten Besatzungstag in Prag zwischen die Kolonnen sowjetischer Militärfahrzeuge ein tschechischer Lastwagen. Er trug ein Plakat, auf dem - in russisch - nur ein einziges Wort stand: WARUM? Dieses Plakat polyglott durch die Geschichte zu fahren, ist die gegenwärtige Aufgabe der Christenheit", schreibt H. D. Bastian in seiner, Theologie der Frage ${ }^{455}$.

Für ein theologisch-journalistisches Selbstverständnis bedeutet dies, als Träger des ,Warums' vor allem anzugehen gegen das wohl am meisten verbreitete Phänomen, die Indifferenz (sprich auch Gleichgültigkeit, Zufriedenheit oder Wohlstandsträgheit). Entscheidend ist, daß auf diese Weise Fragen gestellt werden, die die Wirklichkeit neu in den Blick kommen lassen, die Mut machen zu einem kritisch-konstruktiven Umgang mit und einer Neuorientierung in dieser Wirklichkeit sub specie spei'.

\section{Resümee}

Die herausgearbeiteten Stichworte erheben keinen Anspruch auf Vollständigkeit und verstehen sich in ihrer Akzentuierung als ein möglicher Ansatz eines theologisch-journalistischen Selbstverständnisses, als ein Ansatz, der Anstoß sein will ${ }^{46}$. Bezogen auf die Situation in den Funkhäusern von ARD und ZDF haben die Durchsicht der Literatur und meine Befragungen einzelner Redakteure mir gezeigt, daß es dort sehr wohl so etwas wie ein(ige) theologisch-journalistische Selbstverständnis(se) der mit den Themata ,Religion und Gesellschaft ${ }^{\circ}$ befaßten Journalisten gibt. Dabei kommt es dennoch $\mathrm{zu}$ unterschiedlichen, ja wesentlich verschiedenen Gewichtungen und Konkretionen der jeweiligen Stichworte (so gerade bei den Aspekten ,Kirche' und ,Verkündigung').

Die Großkirchen müssen trotz ihrer regelmäßig wiederkehrenden Klagen ganz sicher nicht um die "Sache des Christlichen" in den Medien bangen. Was allerdings den Kirchen (oder besser gewissen Kreisen in beiden Großkirchen) weniger Anlaß zur Kritik als vielmehr Anstoß zur selbstkritischen Reflektion geben sollte, ist die Tatsache, daß sie als Institutionen mit ihren Forderungen bzw. Wünschen nach Selbstdarstellung sowohl aus theologischen als auch medienspezifischen Gründen immer weniger Erfolg haben werden (und diesbezüglich auch nicht auf die neuen Medien bauen sollten!). Die reine Berichterstattung über Kirche und die besondere Berücksichtigung der beiden großen Konfessionen sind in den Jahrzehnten der Kirchenfunkarbeit in der Nachkriegszeit zurückgetreten zugunsten einer Programmkonzeption, die die eigene Arbeit vor allem als Anwaltschaft in der Gesellschaft für die religiöse Dimension menschlichen Lebens sieht. Die Kirchen sind damit in guter theologischer Tradition hinter die von ihnen verkündete Botschaft zurückgetreten.

Die Chance einer vergleichsweise großen Unabhängigkeit verpflichtet die öffentlichrechtliche Kirchenfunkarbeit nach dem ihr eigenen kritischen Beitrag im Gespräch der Gesellschaft zu fragen. Im Rückgriff auf die von ihm zu vermittelnde Nachricht, die "Gute Nachricht", muß Kirchenfunk in all seinen Aspekten (ob Stellvertretung, Lebenshilfe, Informationsgebung usw.) eher konstruktiv-radikal, d. h. auf die Wurzeln hin, provozieren, denn mit dem Faktischen in Kirche und Gesellschaft konform gehen. Die Redaktionsarbeit gerade im Kirchenfunk hat die Chance, "Atemraum zu schaffen für die öffentliche Meinungsbildung ${ }^{\text {"47 }}$, und zugleich selbst jener Freiraum im Funk zu sein, in dem Fragen und Antwortversuche heranwachsen dürfen, die für den einzelnen ebenso wie für Gesellschaft und Kirche lebensnotwendig sind. 


\section{Anmerkungen}

1 F. Brühl: Kirchenfunksendungen erzielen ein beispielloses Echo. In: „epd/Kirche und Rundfunk", Nr. 4/1972, 26. 1. 72.

2 Diese Arbeit, die angeregt wurde durch meine eigene freie journalistische Mitarbeit im Rahmen der Kirchenfunkarbeit, wurde 1981 an der Phil.-Theol. Hochschule St. Georgen, Frankfurt a. M. eingereicht. Es ging mir darum, Ansätze und Antwortversuche zur Frage eines theologisch-journalistischen Selbstverständnisses in der Kirchenfunkarbeit zu erarbeiten, zu vergleichen und weiterzuentwickeln. Für diese Diplomarbeit, die dem vorliegenden Artikel zugrunde liegt, habe ich neben der einschlägigen Literatur vor allem auf eigene Befragungen von fünf Redakteuren(innen) zu diesem Thema zurüdkgegriffen. Die nadh ihrer Konfession, Sozialisation, Berufserfahrung und inhaltlicher Ausrichtung verschiedenen Redakteure von ARD und ZDF wurden gezielt nach dem Selbstrcrständnis ihrer eigenen Arbeit befragt. Einzelne Gesprächsäußerungen davon finden sich auch in diesem Artikel.

3 Zum ,Fernseh-Urteil' des BVG von 1961 vgl.: Günter Zehner (Hrsg.): Der Fernsehstreit vor dem Bundesverfassungsgericht. Eine Dokumentation des Prozeßmaterials. Karlsruhe 1965 , S. $300-339$.

4 Ebd.

5 Martin Jenke: Gruppen und Programme. Rundfunk als Forum herrschaftsfreier Kommunikation. In: Evgl. Konferenz für Kommunikation (Hrsg.): Rundfunk als machtfreier Raum. Frankfurt a. M. 1972 (medium-dokumentation 1), S. 72. Als interessanten Diskussionsbeitrag zu dieser Debatte über den Vermittlungsauftrag journalistischer Arbeit vgl. auch: Peter Glotz: Journalisten heute. Eine Erinnerung an das Ethos publizistischer Vermittlung. In: „epd/Kirche und Rundfunk" ${ }^{c}$, Nr. 49/1981 v. 1. 7.81.

- Pastoralinstruktion ,Communio et Progressio - Uber die Instrumente der sozialen Kommunikation, kommentiert von Hans Wagner. Trier 1971, S. 213.

7 Was heißt im übrigen "gesellschaftlich-relevant", wann ist eine Gruppe für die Gesamtgesellschaft relevant? Etwa erst dann, wenn die etablierten, herrschenden und sich selbst relevant nennenden Gruppen und Parteien sie als ihresgleichen anerkannt haben? Eine von einigen Journalisten zwar praktizierte Einschätzung, die aber der Wirklichkeit nie gerecht wird.

8 Manfred Linz: Ein Kommentar zum Leben. Uber Religion im Rundfunk. In: „medium“ 4/1967, S. 35.

- Günther Heidtmann: Kirchliche Publizistik. Auftrag - Aufgaben - Aufbau. In: „Evangelische Kommentare $^{\text {" }} 2 / 1969$, S. 384.

10 In Erinnerung an die früheren Diskussionen um das Verhältnis von Verkündigung und Information zueinander sei auf die beiden programmatischen Streitschriften verwiesen: Hans-Eckehard Bahr: Verkündigung als Information. Zur öffentlichen Kommunikation in der demokratischen Gesellschaft. Hamburg 1968; und: Hans-Jürgen Schultz: Information als Verkündigung? In: H. Breit/W. Höhne (Hrsg.): Die provozierte Kirche. München 1968, S. 91-109.

11 Vgl. Hans-Dieter Bastian: Verfremdung und Verkündigung. Gibt es eine theologische Informationstheorie? München 1967, insbes. S. 20-34; ferner dazu: H.-E. Bahr: a. a. O., S. $120 \mathrm{ff}$.

12 H.-J. Schultz: a. a. O., S. 103/106.

13 Hans Bolewski: Information und Kirche. In: "Lutherische Monatshefte" 6/1967, S. 18.

14 Wie selten und umstritten es beispielsweise in den öffentlich-rechtlichen Anstalten ist, dem Zuschauer/Zuhörer konkrete Möglichkeiten aufzuzeigen, bewies u. a. die Auseinandersetzung über die Spendenaufrufe in der Sendung, report' (SWF) von Franz Alt; vgl. dazu den sehr aufschlußreichen Disput zwischen Franz Alt, Carl Weiß und Hendrik Schmidt. In: „epd/Kirche und Rundfunk" Nr. 7/1982, 30. 1. 82.

15 Unter den damals recht zahlreichen Beiträgen zum Thema, direkte-indirekte Verkündigung' sei auf einen sehr interessanten Artikel besonders verwiesen: Heinz Schwitzke: Unzuständige Gedanken eines Liebhabers der Poesie über die Begriffe der ,direkten' und ,indirekten" Verkündigung. In: „medium" $1 / 1964$, S. 169-178 u. 253-264. 
${ }^{16}$ H.-J. Schultz: a. a. O., S. 107.

17 Johann Baptist Metz: Verkündigung und Gesellschaft. Zur Theologie der religiösen Information. In: K. Becker / K. A. Siegel, (Hrsg.): Dynamik der Kommunikation. Frankfurt a. M. 1968, S. 9-20. Das Wort Verkündigung will mehr als nur gehört werden; es will im Verkünden und im Hören bereits etwas vermitteln von seiner befreienden Botschaft: ${ }_{n}$ Das Reden von Gott zielt auf die Vermittlung der Freiheit, die das faktisch Gewordene und Bestehende verändert, zerspielte Freiheit wiedergibt. Das Ziel des Redens ist befreites, veränderndes Handeln. ${ }^{\alpha}$ (Helmut Peukert, Wissenschaftstheorie - Handlungstheorie Fundamentale Theologie. Düsseldorf 1976, S. 61.)

18 Traugott Stählin: Im Dienst der Versöhnung. Theologische Reflexionen zu den Massenmedien. In: ${ }_{n}$ Kunst und Kirche", November 1977, 168-170.

19 J. B. Metz: Politische Theologie. In: „Neues Forum“ 14/1967, S. 13-17.

20 Wer aber sind diese Hoffnungs- und Rechtlosen, Verzweifelten und Unterdrückten? In einer Leistungsgesellschaft wie der bundesdeutschen zeichnet sich zunehmend ab, daß gerade die materielle Privilegierten gar nicht so selten zu einer neuen, ganz anderen Gruppe von "Armen" und Hoffnungslosen werden. Auch hier ist der Kirchenfunk auf eine spezifische Weise gefordert.

${ }^{21}$ Michaela Pilters: Christsein und Journalismus. In: Zum Gespräch verhelfen / Adht Essays, hrsg. vom ,Institut zur Förderung publizistischen Nadhwuchses'. München 1979, S. 29.

${ }^{22}$ Interessant ist in diesem Zusammenhang auch die Beobachtung, daß sich zwar eine stattliche Anzahl ehemaliger protestantischer Pfarrer in den Kirchenfunkredaktionen findet, jedoch katholischerseits kein Geistlicher auf irgendeiner Ebene der öffentl.-rechtl. Medien offiziell angestellt ist. Gründe dafür dürften wohl in den verschiedenen Amtsverständnissen, aber auch in unterschiedlichen gesellschaftspolitischen Konzeptionen zu suchen sein.

23 Wilhelm Schätzler: Zurück zu den Fundamenten. Katholische Medienpolitik, Interview mit W. Schätzler. In: „Rheinischer Merkur“, 11. 5. 79. Zu den verschiedenen Erwartungshaltungen kath. bzw. protest. Kirchenvertreter gegenüber den Kirchenfunkredaktionen vgl. auch die entsprechenden Außerungen in der Fernsehsendung (,Glashaus - TV intern') "Nein und Amen - Kirche im Fernsehen am Beispiel des ZDF", von U. Hoffmann und H. J. Dörger (ARD/WDR, 15. 11. 81); Wortlaut in: „epd/Kirche und Rundfunk", Nr. 89/1981, 18. 11. 81 .

${ }^{24}$ Vgl. zu den verschiedenen Vorwürfen kirchlicher Vertreter das Protokoll des ,Bensberger Spitzengesprächs", zu dem für den 9. 10.72 die ,Gesellschaft kath. Publizisten' geladen hatte; Thema: ,Kirche und Kirchenfunk: Ein notwendiger Konflikt?, Niederschrift und Bericht von Hermann Boventer' (nicht veröffentlicht). Bezüglich der im Artikel erwähnten neueren Vorwürfe kirchlicherseits vgl. die Studie von Gerburg-Elisabeth Vogt: Kirche und Fernsehanstalten. Osnabrück 1978. Frau Vogt bemühte sich dort um eine Fundamentierung derartiger Vorwürfe über den Weg einer kommunikationstheoretischen Aufarbeitung und Analyse einzelner Kirchenfunkbeiträge; so heißt es dort: „Die Verdrängung der offiziellen Kirche aus den Programmen ... setzt sich auf der Ebene der redaktionellen Beiträge fort, indem die Kirche als Institution zugunsten einzelner Gruppen vernachlässigt wird. Kommen die Vertreter der offiziellen Kirche zu Wort, besteht eine deutliche Tendenz, progressive Stimmen zu bevorzugen und die Vermittlung des Ganzen zu vernachlässigen" (S. 153/154).

${ }^{25}$ Konrad Simons: Konflikte im Kirchenfernsehen - z. B. ,Blidkfeld'. In: „Communicatio Socialis" 1979, S. 340-346.

2* Otto B. Roegele: Sinn, Aufgabe und Maßstäbe kirchlicher Sendungen. In: „Funk-Korrespondenz" Nr. 47/1976, 18.11.76.

27 Vgl. ergänzend die Debatte zwischen Manfred Linz und Henry Fischer 1972 in der

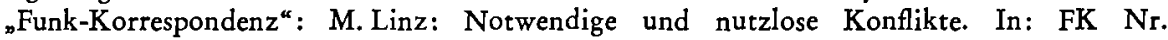
29-30/1972; bzw. H. Fischer: Wahrscheinlich doch ein notwendiger Konflikt. In: FK Nr. $31 / 1972$.

26 Günther Heidtmann: Auswirkungen der Massenmedien auf die Kirche. In: "medium“ 4/1967, S. 94.

- M. Linz: Notwendige und nutzlose Konflikte, S. 3. 
${ }^{30}$ Geht man aus von der Häufigkeit und Intensität, mit der dieses Stichwort seinen Niederschlag in der Literatur findet, so scheint es eindeutig im protestantischen Konzept einer ,christlichen Publizistik' eine stärkere Beachtung zu finden als etwa im katholischen Raum.

31 Robert Geisendörfer: Aufgaben evangelischer Publizistik heute. In: Inge Geisendörfer (Hrsg.): Robert Geisendörfer - Für die Freiheit der Publizistik. Stuttgart/Berlin 1978, S. 40 .

32 Heinrich Fries: Kirche. In: Handbuch theologischer Grundbegriffe, hrsg, von H. Fries in 5 Bänden. München 1970, Bd. II, S. 464 f.

33 Jörg Zink: Was die Medien verschweigen. In: „epd/Kirche und Rundfunk“ Nr. 71/1981, 16. 9.81 .

34 Fast jede Kirchenfunkredakton betreut beispielsweise eine eigene Sendereihe, für die das Stichwort der ,Lebenshilfe' zutrifft.

35 Zum gesamten Stichwort der Lebenshilfe vgl, als Literatur: Internationale Arbeitsgemeinschaft für Kommunikationspädagogik (Hrsg.): Massenmedien und Lebenshilfe. Ludwigshafen 1980; ferner sei verwiesen auf die Referate und Diskussionsbeiträge im Rahmen der ,Hörfunk-Gespräche 1979` des ,Gemeinschaftswerk der evgl. Publizistik' (GEP), Frankfurt a. M. zum Thema: "Orientierungs- und Lebenshilfe im Hörfunk" (Stuttgart, 5. bis 7. Nov. '79).

so T. Stählin: Im Dienst der Versöhnung, S. 169.

37 Michael Albus: ,Kontakte - Magazin für Lebensfragen' - Eine Sendereihe des ZDF. In: Massenmedien und Lebenshilfe, S. 138. Vgl. von demselben Autor: Kirchliche Sendungen im Fernsehen am Beispiel des ZDF - Redaktion Kirche und Leben (kath.), in: "Communicatio Socialis" 1980, S. 23-27.

38 T. Stählin: a. a.O.

39 Vgl. Dieter Stolte: Der Programmauftrag des Rundfunks. In: „Stimmen der Zeit" 1980, S. 75-88; bzw. das epd-Interview mit ihm nach seiner Intendantenwahl. In: „epd/Kirche und Rundfunk “" Nr. 34/1982, 5. 5. 82; dort heißt es u. a.: „... ist es nicht mehr ausreichend zu sagen, was ist, also die Darstellung der Konflikte, die ungeschminkte Widerspiegelung der Probleme ... sondern es scheint mir heute mehr denn je unverzichtbar, auch zu zeigen, was sein könnte."

${ }^{40} \mathrm{H}$. E. Bahr: Lebenserwartungen und Lebenshilfe in unserer Gesellschaft, (noch unveröffentlichtes) Manuskript eines Referates bei den Hörfunk-Gesprächen des GEP 1979 (vgl. Anm. 35), S. 6.

41 Ebd.: S. 8.

42 Zur Diskussion dieses Konzeptes vgl.: Manfred Linz: Ein Kommentar zum Leben; ferner: Cornelius Bormann: Kein Kommentar zum Leben. In: „medium“ 1971, S. 195-197 und (als Replik) M. Linz: Bescheidenheit am falschen Platz. In: „medium“ 1971, S. 198-201.

${ }^{43}$ M. Linz: In der Wolle christlich gefärbt. Interview mit M. Linz. In: „medium " 7/1977, S. $14-16$.

44 Vgl. Fritz P. Schaller: Sind christliche Journalisten moderne Missionare? In: „diakonia 1976, S. 77-83.

45 Hans Dieter Bastian: Theologie der Frage. München 1969, S. 346.

48 Wie bereits eingangs erwähnt, lassen sich ganz sicher auch andere Akzentuierungen und Stichworte denken, wie sie in diesem Artikel zu finden sind.

${ }^{47}$ Helmut Simon im Gespräch mit Heiner Michel über Kirche im Fernsehen. In: „epd/Kirche und Rundfunk", Nr. 32/1982, 28. 4. 82.

\section{SUMMARY}

How do the editors working in the religious departments of two German television channels (A.R.D. and Z.D.F.) themselves see and understand their journalistic role? This was the basic question of a study based on the respective literature and also on personal 
interviews. The results show that there is indeed a specific own conviction of these editors, who are of course expressed by them in different ways. The following are some of the essential headings for their work according to their own understanding: Information, Proclamation, Church, Representation, Assistance and the awareness of the vital responsibilities in the life of society and man.

Also the fact of working in a public service system with certain independency from the official Church give a special sense for responsibility towards the role of the media in public life and public opinion. As for the programme content of the productions of the religious departments in the past decades, the part of the big Churches diminished in favour of a more immediate own concept to serve the religious and existential dimensions of human life at large.

\section{RESUME}

Comment les journalistes travaillant dans les rédactions des émissions religieuses de l'ARD et du ZDF comprennent-ils leurs tâches, - qu'en est-il de leur propre intelligence en matière de journalisme et de théologie? Un examen de cette question à l'aide de littérature et à l'aide de questions appropriées parmi les rédacteurs a montré qu'il existe une intelligence spécifique propre de ces journalistes ce qui est cependant accentué différemment par chacun. En détail, comme aspects et devoirs principaux du travail d'émissions de radio religieuses, il s'en suit les mots de repères programmatiques suivants: "information" / "message" / "Eglise" / "représentation" / "aide vitale“ et la "tenue éveillée de la question concernant l'essentiel dans la vie de chacun et dans celle de la sociétéc.

A cela vient s'ajouter le fait que, pour ce travail de programme, l'organisation juridique et publique joue un rôle très important, c'est-à-dire on travaille indépendamment de l'Eglise et voit en cela sa responsabilité, à savoir étre "medium et facteur" de la formation de l'opinion publique. Dans les décennies passées, le reportage se rapportant aux contenus des programmes des rédactions des émissions de radio religieuses et l'égard particulier rendu aux grandes Eglises se sont retirés en faveur d'un concept de programme qui voit le propre travail surtout comme mandat dans la société pour les dimensions religieuses et existentielles de la vie humaine.

\section{RESUMEN}

Como ven y entienden su rol periodistico los redactores que trabajan en los departamentos de información religiosa de dos canales de televisión alemanes? (ARD y ZDF) Esta fué la principal pregunta de un estudio basado en las publicaciones respectivas y también en entrevistas personales. El resultado muestra que hay de veras una propia y específica convicción en estos redactores que por supuesto son expresados por ellos de diferentes maneras. Los siguientes son algunos de los títulos (lineas ...) esenciales para su trabajo en acuerdo a su propia comprensión: Información, Proclamación, Iglesia, Representación, Asistencia y la certitud de las responsabilidades vitales en la vida de la Sociedad y del Hombre.

También el hecho de trabajar en un sistema de servicio público con una cierta independencia de la Iglesia oficial dá un especial sentido de responsabilidad frente al rol de "medias“ en la vida y opinión públicas.

En relación al contenido programático de las producciones de los departamentos religiosos en las decadas pasadas, la parte de las grandes Iglesias ha disminuido en favor de un concepto más propio e inmediato de servir las dimensiones religiosas y existenciales de la vida humana en general. 